Nominations for Officers of the Canadian Political Science Association

In accordance with the provisions of article VIII,2, of the Constitution of The Canadian Political Science Association, the Nominating Committee invites nominations for the offices of President-Elect; for five Members-at-Large on the Board of Directors; and for three members of the 1981-82 Nominating Committee.

You are reminded that each nomination requires the signatures of two proposers and that it must be accompanied by a statement of acceptance signed by the nominee, as well as by her/his 100 -word curriculum vitae.

All participants in these procedures must of course be paid-up members of the Association. You are further reminded that voting is done by mail ballot.

Nominations must be received by N. H. Chi, Secretary-Treasurer, Canadian Political Science Association, Room 1403, Arts Tower, Carleton University, Ottawa, Ontario K1S 5B6, by January 15, 1981 .

\title{
Nominations à la direction de l'Association canadienne de Science politique
}

Selon l'article VIII,2, de la constitution, le comité de nomination invite les membres de l'Association canadienne de Science politique à présenter des nominations aux postes de président-désigné; conseillers (5) du conseil de direction, et membres (3) du comité de nomination 1981-82.

Il faut que chaque nomination soit accompagnée de deux signataires et d'un avis d'acceptation signé par la personne choisie, ainsi qu'un curriculum vitae de 100 mots.

Tous les participants dans la procédure en cours doivent être membres en règle avec l'Association. Veuillez prendre note que le vote se fera par courrier.

Les nominations doivent parvenir au Secrétaire-trésorier, N. H. Chi, Association canadienne de Science politique, Salle 1403, Arts Tower, Carleton University, Ottawa, Ontario KIS 5B6, avant le 15 janvier, 1981. 